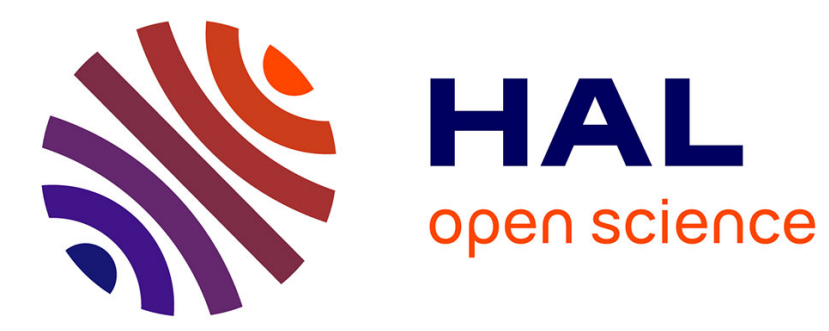

\title{
Exponentially Stable Interval Observers for Linear Systems with Delay.
}

\author{
Frédéric Mazenc, Silviu-Iulian Niculescu, Olivier Bernard
}

\section{To cite this version:}

Frédéric Mazenc, Silviu-Iulian Niculescu, Olivier Bernard. Exponentially Stable Interval Observers for Linear Systems with Delay.. SIAM Journal on Control and Optimization, 2012, 50 (1), pp.286-305. $10.1137 / 100812124$. hal-00761603

\section{HAL Id: hal-00761603 \\ https://hal.inria.fr/hal-00761603}

Submitted on 29 May 2020

HAL is a multi-disciplinary open access archive for the deposit and dissemination of scientific research documents, whether they are published or not. The documents may come from teaching and research institutions in France or abroad, or from public or private research centers.
L'archive ouverte pluridisciplinaire HAL, est destinée au dépôt et à la diffusion de documents scientifiques de niveau recherche, publiés ou non, émanant des établissements d'enseignement et de recherche français ou étrangers, des laboratoires publics ou privés. 


\title{
EXPONENTIALLY STABLE INTERVAL OBSERVERS FOR LINEAR SYSTEMS WITH DELAY*
}

\author{
FRÉDÉRIC MAZENC ${ }^{\dagger}$, SILVIU-IULIAN NICULESCU ${ }^{\ddagger}$, AND OLIVIER BERNARD ${ }^{\S}$
}

\begin{abstract}
This paper focuses on the analysis and design of families of interval observers for linear systems with a pointwise delay. First, it is proved that classical interval observers for systems without delays are not robust with respect to the presence of delays, no matter how small delays are. Next, it is shown that, in general, for linear systems with delay, the classical interval observers endowed with a pointwise delay are unstable. A new type of design of interval observers enabling circumvention of these obstacles is proposed. It provides framers that incorporate distributed delay terms. The proposed interval observers are assessed through a nonlinear biotechnological model.
\end{abstract}

Key words. interval observer, exponential stability, delay

AMS subject classifications. 93C15, 93D20

DOI. $10.1137 / 100812124$

1. Introduction. The interval observer method is a recent state estimation technique, initiated in [7] and extended and applied in many case studies; see, for instance, [23], [28], [1], [24]. It was initially developed for biological systems, whose models are often roughly known but have disturbances, (unknown) initial conditions, parameters, or inputs which belong to known intervals at any instant [4]. More precisely, one can summarize this technique as follows. Consider a nonlinear system

$$
\dot{x}(t)=f_{1}(x(t), w(t))
$$

with $x \in \Re^{n}, w \in \Re^{\ell}$, endowed with the output

$$
y(t)=f_{2}(x(t)) \in \Re^{q},
$$

where $w$ is an unknown time-varying function such that there are two known Lipschitz continuous functions $w^{-}(t), w^{+}(t)$ such that for all $t \geq 0$, the inequalities

$$
w^{-}(t) \leq w(t) \leq w^{+}(t)
$$

hold componentwise. Then the dynamical system given by

$$
\left\{\begin{array}{l}
\dot{z}(t)=g(z(t), \bar{w}(t), y(t)), \\
x^{+}(t)=h^{+}(z(t), \bar{w}(t), y(t)), \\
x^{-}(t)=h^{-}(z(t), \bar{w}(t), y(t)),
\end{array}\right.
$$

with $\bar{w}=\left(\left(w^{+}\right)^{\top},\left(w^{-}\right)^{\top}\right)^{\top} \in \Re^{2 \ell}, g: \Re^{n_{z}} \times \Re^{2 \ell} \times \Re^{q} \mapsto \Re^{n_{z}}$, and $h: \Re^{n_{z}} \times \Re^{2 \ell} \times \Re^{q} \mapsto$ $\Re^{2 n}, h=\left(\left(h^{+}\right)^{\top},\left(h^{-}\right)^{\top}\right)^{\top}$ defines a framer if for any initial conditions $x(0) \in \Re^{n}$, $z(0) \in \Re^{n_{z}}$, such that the inequalities

$$
x_{0}^{-} \leq x(0) \leq x_{0}^{+}
$$

* Received by the editors October 18, 2010; accepted for publication (in revised form) November 3, 2011; published electronically January 19, 2012.

http://www.siam.org/journals/sicon/50-1/81212.html

${ }^{\dagger}$ Projet INRIA-Saclay DISCO, CNRS-Supelec, 3 rue Joliot Curie, 91192, Gif-sur-Yvette, France (Frederic.MAZENC@lss.supelec.fr).

${ }^{\ddagger}$ Laboratoire des Signaux et Systèmes, CNRS-Supélec, 3 rue Joliot Curie, 91192 Gif-sur-Yvette, France (Silviu.Niculescu@lss.supelec.fr).

${ }^{\S}$ EPI COMORE INRIA Sophia-Antipolis, 2004 route des Lucioles, BP 93, 06902 Sophia-Antipolis, France (olivier.bernard@inria.fr). 
with $x_{0}^{ \pm}=h^{ \pm}(z(0), \bar{w}(0), y(0))$, are satisfied, then the solutions of (1.1) and (1.4) with $x(0)$ and $z(0)$, respectively, as initial condition satisfy, componentwise, the inequalities

$$
x^{-}(t) \leq x(t) \leq x^{+}(t)
$$

for all $t \geq 0$. Moreover, if (1.4) is globally asymptotically stable when $\bar{w}$ is identically equal to zero, it is called an interval observer. It is worth mentioning that the interval observers encountered in the literature are frequently designed for cooperative and globally asymptotically stable systems (see, e.g., [31] for the corresponding definition or section 2 of the present paper), allowing a simplified design [4]. Such interval observers for system (1.1) are then of the following type (to simplify the presentation we assume here that $\frac{D f_{1}}{D w}$ is a nonnegative matrix and that there is no output):

$$
\left\{\begin{array}{l}
\dot{z}^{+}(t)=f_{1}^{+}\left(z^{+}(t), \bar{w}(t)\right), \\
\dot{z}^{-}(t)=f_{1}^{-}\left(z^{-}(t), \underline{w}(t)\right), \\
x^{+}(t)=z^{+}(t), \\
x^{-}(t)=z^{-}(t) .
\end{array}\right.
$$

When a globally asymptotically stable system is not cooperative, interval observers can be constructed, provided the system can be transformed through a change of coordinates into a cooperative system (see, for instance, $[25,20]$ ). This remark opens a lot of perspectives in constructing interval observers, which are of interest but beyond the scope of our work. However, it is important to keep in mind that for any globally Lipschitz system with additive disturbances, framers can always be constructed [25], but not necessarily stable framers.

The success of this state estimation method is mostly due to three reasons. First, the method applies and gives satisfactory results when systems with large uncertainties are studied. Second, this technique takes advantage of an approximate knowledge of the initial condition and gives guaranteed information on the unknown state of the system studied at any instant $t \geq 0$ of time, whereas classical observers only provide a useful information asymptotically, that is, from an engineering point of view, for sufficiently large values of time. Third, as explained in [7] and [19], interval observers can be used in combination with classical observers to obtain an upper bound of the distance between the real state of the system and the values predicted by one of its observers.

The interval observers available in the literature are all made for systems represented by ordinary differential equations. Since delays are encountered in many dynamical systems, especially in biological systems (see, e.g., $[8,22,11]$ and the references therein), arising as an intrinsic part of the system, through input and measurement channels, feedback, and propagation of some signals, or as a result of a simplification of partial differential equations, it is appealing to investigate if interval observers can be used for systems with delays. To the best of the authors' knowledge, this problem has never been addressed, although some results on the applications of interval analysis techniques [15] to delay systems have been discussed in the literature (see, for instance, [17]). Let us recall that in contrast, constructing observers for systems with delay has been a subject of recurring interest since the 1970s (see, e.g., [16, Chapter 3], [2, 29, 5, 27, 3, 30] and the references therein).

Since the interval observer technique allows us to cope with poorly known systems, one may expect this technique to be robust with respect to the presence of a small delay in the equations of the observed system: indeed, in some cases, a small delay can 
be considered as a small perturbation which does not significantly modify the behavior of a system. (For further discussions on such topics, see, for instance, [22] and the references therein.) In the first part of the present work, we will show that this is not the case for systems endowed with exponentially stable linear interval observers. In spite of what intuition may suggest, the classical interval observers without delay in general do not work when applied to exponentially stable linear systems with a pointwise delay, no matter how small this delay is. Overcoming this obstacle, that is, determining interval observers that give a satisfactory estimation result when a small delay is present, is not a trivial task. In particular, we will show that a direct adaptation of the classical interval observer construction technique to the case of systems with delay leads, in general, to unstable framers with pointwise delays. This is a serious drawback because unstable framers no longer provide valuable estimates when the time becomes large. It is worth noting that the technique of the proofs of this first part have some similarities with those of the contributions [11, section 3], [13, section III] and use [12, Proposition 3.2].

In the second part of the paper, for a general family of exponentially stable linear systems with a pointwise delay, we propose a new construction of interval observers which converge exponentially to the origin when no disturbance is acting. The key feature of the framers we introduce is that their dynamics incorporate distributed delay terms, although they are framers for systems with pointwise delays. Their construction is based on a classical model transformation of the original system which relies on an appropriate integration over one delay interval. The advantages and the drawbacks of using such a technique were largely discussed in the literature (see, for instance, $[26,8,6,9]$ and the references therein). It gives a representation of the system's dynamics as "pieces of trajectories." The benefit of this representation is that it makes it possible to design for the original system exponentially stable interval observers of a new type that we shall call " $k$-framers," where $k$ is any integer larger than 1. As we shall see, there is an important difference between 1-framers and $k$ framers with $k \geq 2$ (see section 2). On the one hand, the notion of a 1 -framer is a direct extension of the notion of "classical" framers designed for systems without delay, to the case of systems with delay. On the other hand, the notion of $k$-framers with $k \geq 2$ is a more sophisticated and perhaps more difficult to understand adaptation of the notion of framers to systems with delays. It allows us to constructed framers possessing the desirable exponential stability property for wide families of systems with delays which do not admit a stable 1-framer. In what follows, particular attention will be paid to 1and 2-framers, which complement each other: 1-framers have the advantage of being simple and 2-framers have the advantage of being applicable to very general families of systems with delay.

Finally, throughout sections 3 and 4 we will consider, for the sake of simplicity, linear systems without specifying whether they are endowed with an output. However, it is important to bear in mind that our results can be fruitfully applied to timedelay systems with output that admit classical linear observers and, in the absence of disturbances, lead to error equations of the form

$$
\dot{e}(t)=A_{1} e(t)+A_{2} e(t-\tau) .
$$

Indeed, what we said earlier about systems endowed with classical observers without delay is also true when a delay is present in the dynamics and/or in the output of a system.

The present paper is an extended version of the preliminary work [21]. The rest of the paper is organized as follows. Section 2 gives definitions and a basic result in 
preparation for the subsequent results. Section 3 focuses mostly on the lack of robustness of standard interval observers with respect to small delays and the limitations of the interval observers with a pointwise delay. Section 4 includes the main results and an illustrative example. An application of the main construction of the paper is presented in section 5. Conclusions are given in section 6 .

\section{Notations, definitions.}

Classical notation and definitions.

- The standard Euclidean norm and the essential supremum are denoted, respectively, $\|\cdot\|$ and $|\cdot|_{\infty}$. $\mathcal{C}_{n}(\mathcal{I})$.

- The class of the continuous $\Re^{n}$-valued functions on an interval $\mathcal{I}$ is denoted

- Throughout the paper, we assume that the functions encountered are sufficiently smooth, unless otherwise stated.

- The argument of the functions will be omitted or simplified whenever no confusion can arise from the context.

- Let $\tau$ be a positive real number. Then, for a given $t \geq 0, x_{t}(\cdot)$ denotes the restriction of $x(\cdot)$ to the interval $[t-\tau, t]$ translated to $[-\tau, 0]$, i.e., $x_{t}(\theta)=x(t+\theta)$, for all $\theta \in[-\tau, 0]$. The solution of a time-delay system described by a functional differential equation (FDE),

$$
\dot{\mathcal{X}}(t)=\mathcal{F}\left(\mathcal{X}_{t}, w_{t}\right)
$$

with an initial condition $\phi_{\mathcal{X}} \in \mathcal{C}_{n}([-\tau, 0])$ at $t_{0}$, will be denoted by $\mathcal{X}(t)$ (instead of $\mathcal{X}\left(t, t_{0}, \phi_{\chi}\right)$ as rigorously done in $[14$, Chapter 2$\left.]\right)$.

- In what follows, all the inequalities must be understood componentwise, i.e., $v=\left(v_{1}, \ldots, v_{r}\right)^{\top} \in \Re^{r}$ and $w=\left(w_{1}, \ldots, w_{r}\right)^{\top} \in \Re^{r}$ are such that $v \leq w$ if and only if for all $i \in\{1, \ldots, r\}, v_{i} \leq w_{i}$.

- If $M$ is a matrix with the entries $m_{i, j}$, then we denote by $M^{+}$(resp., $M^{-}$) the matrix whose entries are $\max \left\{0, m_{i, j}\right\}$ (resp., $\min \left\{0, m_{i, j}\right\}$ ).

- A square matrix is said to be cooperative or essentially nonnegative if its offdiagonal terms are nonnegative. A system $\dot{x}=f(x, w)$ is said to be cooperative if the Jacobian matrix of $f$ with respect to $x$, denoted $\frac{D f}{D x}(x, w)$, is a cooperative matrix for all $(x, w)$.

- We shall use the following definitions [12].

Definition 2.1. Consider an FDE

$$
\dot{\mathcal{X}}(t)=\mathcal{F}\left(\mathcal{X}_{t}\right)
$$

with $\tau \geq 0$, where $\mathcal{F}: \mathcal{C}_{n}([-\tau, 0]) \mapsto \Re^{n}$ is a locally Lipschitz function.

The system (2.1) is said to be nonnegative if for every initial condition $\phi_{\mathcal{X}} \in$ $\mathcal{C}_{n}([-\tau, 0]), \phi_{\mathcal{X}}(t) \geq 0$ for all $t \in[-\tau, 0]$, the solution $\mathcal{X}(t)$ to $(2.1)$ is nonnegative over $[-\tau,+\infty)$.

- We also recall [12, Proposition 3.2], which will be instrumental in our analyzes and constructions of framers.

Proposition 2.2. Consider the linear time-invariant system

$$
\dot{\mathcal{X}}(t)=A \mathcal{X}(t)+A_{d} \mathcal{X}(t-\tau)
$$

with $\tau \geq 0$. This system is nonnegative if and only if the matrix $A$ is essentially nonnegative and the matrix $A_{d}$ is nonnegative.

Copyright $@$ by SIAM. Unauthorized reproduction of this article is prohibited. 
Remark 1. To give insight into the interplay between the notions of cooperative and nonnegative systems, we observe that in the particular case of linear systems with a pointwise delay, Proposition 2.2 implies that if the system (2.2) is nonnegative, then necessarily the system $\dot{\mathcal{X}}(t)=A \mathcal{X}(t)+w(t)$ is cooperative.

Definitions of framers and interval observers. As indicated in the introduction, two ingredients are essential in the definition of framers: (i) the existence of functions $w^{+}$and $w^{-}$that "bound" the disturbance $w$ on the whole time-domain interval, and (ii) an appropriate trajectory-based interpretation of the dynamics. Based on this observation, there are several possibilities to extend the notion of framer to time-delay systems, and in fact these extensions depend on the way the notion of "state" is interpreted and used in the FDE setting. This leads us to the definition of $k$-framer.

Definition 2.3 (k-framer). Consider an FDE

$$
\dot{\mathcal{X}}(t)=\mathcal{F}\left(\mathcal{X}_{t}, w_{t}\right)
$$

with $\mathcal{F}: \mathcal{C}_{n}([-\tau, 0]) \times \mathcal{C}_{\ell}([-\tau, 0]) \mapsto \Re^{n}$ locally Lipschitz and assume that there are two known Lipschitz continuous functions $w^{-}(t), w^{+}(t)$ such that for all $t \geq-\tau$,

$$
w^{-}(t) \leq w(t) \leq w^{+}(t),
$$

and such that for all initial conditions, the solutions of (2.3) are defined over $[-\tau,+\infty)$. Then, the dynamical system

$$
\left\{\begin{aligned}
\dot{\mathcal{Z}}(t) & =\mathcal{G}\left(\mathcal{Z}_{t}, \bar{w}_{t}\right), \\
\mathcal{X}^{+}(t) & =\mathcal{H}^{+}(\mathcal{Z}(t)), \\
\mathcal{X}^{-}(t) & =\mathcal{H}^{-}(\mathcal{Z}(t))
\end{aligned}\right.
$$

with $\bar{w}=\left(\left(w^{+}\right)^{\top},\left(w^{-}\right)^{\top}\right)^{\top} \in \Re^{2 \ell}, \mathcal{G}: \mathcal{C}_{n_{z}}([-\tau,(k-1) \tau]) \times \mathcal{C}_{2 \ell}([-\tau,(k-1) \tau]) \mapsto \Re^{n_{z}}$ a locally Lipschitz function, $k \geq 1$ an integer, $\mathcal{H}: \Re^{n_{z}} \mapsto \Re^{2 n}, \mathcal{H}=\left(\left(\mathcal{H}^{+}\right)^{\top},\left(\mathcal{H}^{-}\right)^{\top}\right)^{\top}$ a continuous function is called a $k$-framer if, for any functions $\phi_{\mathcal{X}} \in \mathcal{C}_{n}([-\tau, 0])$ and $\phi_{\mathcal{Z}} \in \mathcal{C}_{n_{z}}([-\tau,(k-1) \tau])$ such that the inequalities

$$
\phi_{\mathcal{X}^{-}}(t) \leq \mathcal{X}(t) \leq \phi_{\mathcal{X}^{+}}(t) \forall t \in[-\tau,(k-1) \tau]
$$

with

$$
\phi_{\mathcal{X}^{-}}(t)=\mathcal{H}^{-}\left(\phi_{\mathcal{Z}}(t)\right), \phi_{\mathcal{X}+}(t)=\mathcal{H}^{+}\left(\phi_{\mathcal{Z}}(t)\right)
$$

are satisfied, then, for all $t \geq(k-1) \tau$, the solution of (2.5) exists and satisfies

$$
\mathcal{X}^{-}(t) \leq \mathcal{X}(t) \leq \mathcal{X}^{+}(t)
$$

Instead of introducing only the definition of 1-framer, we introduced the wider definition of $k$-framer because it provides with a wide variety of interval observers associated with specific initial conditions. They can be found in most of the cases. In particular, constant initial conditions can always be found for the important family of systems (2.3) such that there exists a constant $m>0$ such that the inequality

$$
\left|\mathcal{F}\left(\phi_{x}, \phi_{w}\right)\right| \leq m\left(1+\left|\phi_{x}(0)\right|+\left|\phi_{x}(-\tau)\right|+\left|\phi_{w}\right|\right)
$$

Copyright (c) by SIAM. Unauthorized reproduction of this article is prohibited. 
is satisfied for all function $\phi_{x} \in \mathcal{C}_{0}([-\tau, 0])$ and all function $\phi_{w}$ continuous over $[-\tau,(k-1) \tau]$. Indeed, consider a solution of the system (2.3) with $\phi_{\mathcal{X}}$ as initial condition. The property (2.9) leads to the inequality

$$
|\dot{\mathcal{X}}(t)| \leq m\left(1+|\mathcal{X}(t)|+|\mathcal{X}(t-\tau)|+\left|w^{+}(t)\right|+\left|w^{-}(t)\right|\right),
$$

from which one can deduce through an integration that for all $t \in[0, \tau]$,

$$
\begin{aligned}
|\mathcal{X}(t)| & \leq|\mathcal{X}(0)|+m \int_{0}^{t}\left(1+|\mathcal{X}(\ell)|+|\mathcal{X}(\ell-\tau)|+\left|w^{+}(\ell)\right|+\left|w^{-}(\ell)\right|\right) d \ell \\
& \leq\left|\phi_{\mathcal{X}}\right|+m \int_{0}^{t}\left(1+|\mathcal{X}(\ell)|+\left|\phi_{\mathcal{X}}(\ell-\tau)\right|+\left|w^{+}(\ell)\right|+\left|w^{-}(\ell)\right|\right) d \ell \\
& \leq m \tau+(1+m \tau)\left|\phi_{\mathcal{X}}(0)\right|_{\infty}+m \int_{0}^{\tau}\left(\left|w^{+}(\ell)\right|+\left|w^{-}(\ell)\right|\right) d \ell+m \int_{0}^{t}(|\mathcal{X}(\ell)| d \ell .
\end{aligned}
$$

It follows from the Gronwall-Bellman inequality [10] that for all $t \in[0, \tau]$,

$$
|\mathcal{X}(t)| \leq b_{1}
$$

with $b_{1}=\left[m \tau+(1+m \tau)\left|\phi_{\mathcal{X}}(0)\right|_{\infty}+m \int_{0}^{\tau}\left(\left|w^{+}(\ell)\right|+\left|w^{-}(\ell)\right|\right) d \ell\right] e^{m}$. Next, by induction and arguing as we did, one can determine bounds $b_{i}$ for $|\mathcal{X}(t)|$ on any intervals $[i \tau,(i-$ $1) \tau]$, where $i$ is an integer. Then the inequalities (2.6) are satisfied with the constant initial conditions

$$
\phi_{\mathcal{X}^{+}}(t)=\sup _{j \in\{1, \ldots, k-1\}}\left\{b_{j}\right\}(1 \ldots 1)^{\top}, \phi_{\mathcal{X}^{-}}(t)=-\phi_{\mathcal{X}^{+}}(t) .
$$

The following definition makes precise the notion of exponentially stable interval observer.

DeFinition 2.4. A k-framer

$$
\left\{\begin{aligned}
\dot{\mathcal{Z}}(t) & =\mathcal{G}\left(\mathcal{Z}_{t}, \bar{w}_{t}\right) \\
\mathcal{X}^{+}(t) & =\mathcal{H}^{+}(\mathcal{Z}(t)) \\
\mathcal{X}^{-}(t) & =\mathcal{H}^{-}(\mathcal{Z}(t))
\end{aligned}\right.
$$

for the system (2.3) is an exponentially stable interval observer if there exists a real number $c>0$ such that any solution of (2.10) with $\bar{w}$ identically equal to zero satisfies

$$
\lim _{t \rightarrow+\infty} e^{c t}\left[\mathcal{X}^{+}(t)-\mathcal{X}^{-}(t)\right]=0
$$

3. Limitations of framers without delay or with a pointwise delay. In this section, we show that in general, interval observers used for exponentially stable linear systems in the absence of delay are not robust with respect to an arbitrarily small delay in the dynamics. We also show that for some exponentially stable systems with an arbitrarily small pointwise delay, determining an exponentially stable interval observer with a pointwise delay only is an open problem (which probably admits no solution). Our results are established for the very simple FDE

$$
\dot{x}(t)=-x(t-\tau)
$$

with $x \in \Re, \tau \geq 0$, and no output, which admits the origin as an exponentially stable equilibrium point, provided $\tau \in\left[0, \frac{\pi}{2}\right)$. However, we wish to point out that they can 
be extended to wide families of linear systems. Finally, this section ends with a result of construction of an exponentially stable 1-framer for a particular family of systems with an arbitrary pointwise delay.

3.1. A nonrobust interval observer. When $\tau=0$, the system (3.1) is cooperative. In that case, it is well known that two copies of the system compose a framer: the system

$$
\left\{\begin{array}{l}
\dot{z}^{+}(t)=-z^{+}(t), \\
\dot{z}^{-}(t)=-z^{-}(t), \\
x^{+}(t)=z^{+}(t), \\
x^{-}(t)=z^{-}(t)
\end{array}\right.
$$

is such that if $x^{-}(0) \leq x(0) \leq x^{+}(0)$, then $x^{-}(t) \leq x(t) \leq x^{+}(t)$ for all $t \geq 0$. Moreover this framer is exponentially stable: all the solutions of (3.2) converge exponentially to the origin when the time goes to infinity.

When $\tau>0$, the behavior of the equations is tremendously different: the system (3.2) is no longer a framer for the system (3.1) in any reasonable sense. More precisely, we have the following result.

Proposition 3.1. For any integer $k \geq 1$, the system (3.2) is not a $k$-framer of (3.1) when $\tau \in\left(0, \frac{\pi}{2}\right)$.

Proof. To prove Proposition 3.1, we proceed by contradiction. Let $\tau \in\left(0, \frac{\pi}{2}\right)$ and assume that there exists an integer $k \geq 1$ such that the system (3.2) is a $k$-framer for the system (3.1). For the system (3.1), we choose an initial condition $\phi_{x}$ such that there exists $t_{s} \in(-\tau, 0)$ such that $\phi_{x}\left(t_{s}\right) \neq 0$. For the $z^{+}$-subsystem and the $z^{-}$subsystem of (3.2), we choose $\phi_{z^{+}}(t)=\phi_{z^{-}}(t)=x(t)$ for all $t \in[-\tau,(k-1) \tau]$. From the definition of $k$-framer and (3.2), it follows that for all $t \geq(k-1) \tau, z^{+}(t)=z^{+}((k-$ 1) $\tau) e^{-t+(k-1) \tau}$ and $z^{+}(t)=z^{-}(t)=x(t)$. Therefore $x(t)=z^{+}((k-1) \tau) e^{-t+(k-1) \tau}$ for all $t \geq(k-1) \tau$. By taking the time derivative of $x(t)$, we deduce from (3.1) that for all $t \geq k \tau$

$$
-z^{+}((k-1) \tau) e^{-t+(k-1) \tau}=-z^{+}((k-1) \tau) e^{-t+k \tau}
$$

We deduce that necessarily $z^{+}((k-1) \tau)=0$. It follows that for all $t \geq(k-1) \tau$, $x(t)=0$. We deduce easily that necessarily $\phi_{x}(t)=0$ for all $t \in[-\tau, 0]$. This fact and our choice of initial condition give a contradiction.

3.2. Framers with pointwise delay. The next natural question which arises is whether there exist exponentially stable interval observers with the pointwise delay $\tau$ for the system (3.1). We have no answer to this general question. However, we show below that two simple adaptations of the framer (3.2) do not work. The first dynamic extension we propose is not a framer and the second is not stable.

First choice. Consider the system

$$
\left\{\begin{array}{l}
\dot{z}^{+}(t)=-z^{+}(t-\tau) \\
\dot{z}^{-}(t)=-z^{-}(t-\tau), \\
x^{+}(t)=z^{+}(t) \\
x^{-}(t)=z^{-}(t)
\end{array}\right.
$$


which is composed of two copies of (3.1) and is deduced from (3.2) by the introduction of $\tau$ in the right-hand side. Let us prove that for any integer $k \geq 1$, this system is not a $k$-framer for the system (3.1). Let $T_{k}=(k-1) \tau$ and let the initial conditions of (3.3) be $\phi_{z^{+}}(t)=-t+T_{k}, \phi_{z^{-}}(t)=0$ for all $t \in\left[-\tau, T_{k}\right]$. Then $\phi_{z^{+}}\left(T_{k}\right)-\phi_{z^{-}}\left(T_{k}\right)=0$, and

$$
\dot{z}^{+}\left(T_{k}\right)-\dot{z}^{-}\left(T_{k}\right)=-\phi_{z^{+}}\left(T_{k}-\tau\right)=-\tau<0 .
$$

It follows that there exists $T_{L}>T_{k}$ such that $z^{+}\left(T_{L}\right)<z^{-}\left(T_{L}\right)$. This allows us to conclude.

Second choice. We may reasonably decide to regard $x(t-\tau)$ as a variable different from $x(t)$, and then the classical strategy of design of framers leads us to consider the FDE

$$
\left\{\begin{array}{l}
\dot{z}^{+}(t)=-z^{-}(t-\tau) \\
\dot{z}^{-}(t)=-z^{+}(t-\tau) \\
x^{+}(t)=z^{+}(t) \\
x^{-}(t)=z^{-}(t)
\end{array}\right.
$$

With the notation $\tilde{x}^{+}=x^{+}-x, \tilde{x}^{-}=x-x^{-}$, we obtain

$$
\left\{\begin{array}{l}
\dot{\tilde{x}}^{+}(t)=\tilde{x}^{-}(t-\tau), \\
\dot{\tilde{x}}^{-}(t)=\tilde{x}^{+}(t-\tau) .
\end{array}\right.
$$

According to Proposition 2.2 any solution of (3.5) with nonnegative initial conditions is nonnegative for all $t \geq-\tau$. It follows that the inequalities

$$
x^{-}(t) \leq x(t) \leq x^{+}(t)
$$

are satisfied for all $t \geq 0$, which implies that (3.4) is a 1-framer for (3.1). However, the system (3.5) is unstable: almost all its solutions go to infinity when the time goes to infinity. Therefore the system (3.4) is not an exponentially stable interval observer. Furthermore, we have the following result. (See Appendix A for the proof.)

Proposition 3.2. The system (3.1) does not possess an exponentially stable 1-framer of the form

$$
\left\{\begin{array}{l}
\dot{Z}(t)=F_{1} Z(t)+F_{2} Z(t-\tau), \\
x^{+}(t)=H_{1} Z(t) \\
x^{-}(t)=H_{2} Z(t)
\end{array}\right.
$$

where $Z \in \Re^{n_{Z}}, n_{Z} \in N, n_{Z} \geq 1$ is arbitrary and $F_{i}, H_{i}(i=1,2)$ are matrices of appropriate dimensions.

In contrast with Proposition 3.2, the result below shows that in particular cases, the presence of a pointwise delay does not preclude from constructing of exponentially stable 1-framers with a pointwise delay.

Proposition 3.3. Consider the system with a pointwise delay

$$
\dot{x}(t)=A x(t)+A_{d} x(t-\tau)
$$

Copyright (c) by SIAM. Unauthorized reproduction of this article is prohibited. 
where the matrix $A$ is essentially nonnegative and the matrix $A_{d}$ is nonnegative. Assume that this system is exponentially stable. Then the system

$$
\left\{\begin{array}{l}
\dot{z}^{+}(t)=A z^{+}(t)+A_{d} z^{+}(t-\tau), \\
\dot{z}^{-}(t)=A z^{-}(t)+A_{d} z^{-}(t-\tau), \\
x^{+}(t)=z^{+}(t), \\
x^{-}(t)=z^{-}(t)
\end{array}\right.
$$

is exponentially stable interval observer for (3.8).

Proof. Let $\tilde{x}^{+}=x^{+}-x$ and $\tilde{x}^{-}=x-x^{-}$. Then

$$
\left\{\begin{array}{l}
\dot{\tilde{x}}^{+}(t)=A \tilde{x}^{+}(t)+A_{d} \tilde{x}^{+}(t-\tau), \\
\dot{\tilde{x}}^{-}(t)=A \tilde{x}^{-}(t)+A_{d} \tilde{x}^{-}(t-\tau) .
\end{array}\right.
$$

Proposition 2.2 implies that the system (3.10) is nonnegative. This allows us to conclude.

Remark 2. For the sake of clarity, Proposition 3.3 is devoted to systems having no disturbances. Immediate extensions to cases where additive disturbances are present can be obtained.

4. Construction of framers for linear systems with delay. In the previous section, we listed three difficulties which arise when one aims at constructing interval observers for linear systems with delay. In this section, we show how these obstacles can be overcome for a large family of linear systems with a pointwise delay (which contains the system (3.1)) by constructing framers of a new type. The key features of these framers is that they have distributed terms in their equations and are exponentially stable, when the delay is smaller than some upper bound. These interval observers are 2 -framers.

4.1. Main result. We consider an FDE with a single delay $\tau \geq 0$,

$$
\dot{x}(t)=A x(t)+B x(t-\tau)+w(t)
$$

with $x \in \Re^{n}, w \in \Re^{n}$ and where $A$ and $B$ are constant square matrices and $w$ is a Lipschitz continuous function. Let $a_{i, j}$ (resp., $b_{i, j}$ ) denote the entries of $A$ (resp., $B$ ) and let

$$
p=\max \left\{\max _{i, j}\left\{\left|a_{i, j}\right|\right\}, \max _{i, j}\left\{\left|b_{i, j}\right|\right\}\right\} .
$$

Let $D$ denote the diagonal matrix such that all the diagonal terms of the matrix $-D+A+B$ are equal to zero and

$$
E=A+B-D \quad \text { and } \quad E_{P}=D+E^{+} .
$$

(See section 2 for the definitions of $E^{+}$and $E^{-}$.) We now introduce the following assumptions.

Assumption 1. The matrix

$$
M=\left[\begin{array}{cc}
E_{P} & -E^{-} \\
-E^{-} & E_{P}
\end{array}\right]
$$

is Hurwitz. 
Assumption 2. Two Lipschitz continuous functions $w^{+}, w^{-}$such that for all $t \geq-\tau$, the inequalities

$$
w^{-}(t) \leq w(t) \leq w^{+}(t)
$$

hold are known.

Observe for later use that Assumption 1 guarantees that there exists a matrix $Q=Q^{\top}>0$ such that

$$
Q M+M^{\top} Q=-I_{n},
$$

where $I_{n}$ denotes the $n \times n$ identity matrix.

We are ready to state and prove the main result of the section.

Theorem 4.1. Assume that the system (4.1) satisfies Assumptions 1 and 2. Then a linear 2-framer can be constructed for this system. This 2-framer is an exponentially stable interval observer when the delay is such that

$$
\tau \in\left[0, \frac{1}{16 n^{2} p^{2}\|Q\|}\right] .
$$

\section{Discussion of Theorem 4.1.}

- We have chosen to study linear systems with an additive disturbance $w(t)$. Our motivation stems from the importance of systems of this type. Observe that other types of disturbances can be considered. For instance, the case where the entries of $A$ or $B$ are not well known may be of interest, but this is beyond the scope of the present work.

- In the particular case where the matrix $A+B$ is cooperative and Hurwitz, $E_{P}=A+B$ and $E^{-}=0$. Thus Assumption 1 is automatically satisfied.

- Assumption 1 is satisfied if and only if the system

$$
\left\{\begin{array}{l}
\dot{r}_{1}(t)=E_{P} r_{1}(t)-E^{-} r_{2}(t) \\
\dot{r}_{2}(t)=E_{P} r_{2}(t)-E^{-} r_{1}(t)
\end{array}\right.
$$

is an exponentially stable system. From this fact, one can deduce that

$$
\left\{\begin{array}{l}
\dot{z}^{+}(t)=E_{P} z^{+}(t)+E^{-} z^{-}(t) \\
\dot{z}^{-}(t)=E_{P} z^{-}(t)+E^{-} z^{+}(t) \\
x^{+}(t)=z^{+}(t) \\
x^{-}(t)=z^{-}(t)
\end{array}\right.
$$

is an exponentially stable interval observer for system (4.1) when $\tau=0$ and $w(t)=0$ for all $t \geq 0$. This result implies that $A+B$ is a Hurwitz matrix.

- The procedure applied to determine 2-framers for the system (4.1) is based on a model transformation over one delay interval. By performing model transformations over $k-1$ delay intervals where $k$ is an integer larger than 1 (see [9] for more details on these transformations), we would be led to the construction of $k$-framers. For the sake of simplicity, we did not investigate the advantages and the drawbacks of this potential result.

4.2. Proof of Theorem 4.1. Let us introduce the notation

$$
\begin{aligned}
\nu_{j}(t) & =\int_{t-\tau}^{t} x_{j}(\ell) d \ell, \nu=\left(\nu_{1}, \ldots, \nu_{n}\right)^{\top}, \\
W(t) & =\int_{t-\tau}^{t} w(\ell) d \ell,
\end{aligned}
$$

Copyright (c) by SIAM. Unauthorized reproduction of this article is prohibited. 
and $G=-B A, H=-B^{2}$. For all $t \geq 0$, the system (4.1) rewrites as

$$
\dot{x}(t)=(D+E) x(t)-B[x(t)-x(t-\tau)]+w(t) .
$$

We deduce that for all $t \geq \tau$, the solutions of the system (4.1) satisfy

$$
\dot{x}(t)=(D+E) x(t)+G \nu(t)+H \nu(t-\tau)+w(t)-B W(t)
$$

or, equivalently,

$$
\begin{aligned}
\dot{x}(t)= & \left(E_{P}+E^{-}\right) x(t)+G^{+} \nu(t)+G^{-} \nu(t)+H^{+} \nu(t-\tau) \\
& +H^{-} \nu(t-\tau)-B^{+} W(t)-B^{-} W(t)+w(t) .
\end{aligned}
$$

This system leads us to consider for the system (4.1) the candidate interval observer

$$
\left\{\begin{aligned}
\dot{z}^{+}(t)= & E_{P} z^{+}(t)+E^{-} z^{-}(t)+H^{+} \nu^{+}(t-\tau)+H^{-} \nu^{-}(t-\tau) \\
& +G^{+} \nu^{+}(t)+G^{-} \nu^{-}(t)+w^{+}(t)-B^{+} W^{-}(t)-B^{-} W^{+}(t) \\
\dot{z}^{-}(t)= & E_{P} z^{-}(t)+E^{-} z^{+}(t)+H^{+} \nu^{-}(t-\tau)+H^{-} \nu^{+}(t-\tau) \\
& +G^{+} \nu^{-}(t)+G^{-} \nu^{+}(t)+w^{-}(t)-B^{+} W^{+}(t)-B^{-} W^{-}(t) \\
x^{+}(t)= & z^{+}(t) \\
x^{-}(t)= & z^{-}(t)
\end{aligned}\right.
$$

with $\nu_{j}^{ \pm}(t)=\int_{t-\tau}^{t} z_{j}^{ \pm}(\ell) d \ell, W^{ \pm}(t)=\int_{t-\tau}^{t} w^{ \pm}(\ell) d \ell, \nu^{ \pm}=\left(\nu_{1}^{ \pm}, \ldots, \nu_{n}^{ \pm}\right)^{\top}$. To show that the system (4.14) is a 2-framer for the system (4.1), we consider for (4.1) an initial condition $\phi_{x} \in \mathcal{C}_{n}([-\tau, 0])$ which, due to the linearity of (4.1), generates for all $t \geq-\tau$ a solution $x(t)$ and, for (4.14), an initial condition $\phi_{z}=\left(\left(\phi_{z^{+}}\right)^{\top},\left(\phi_{z^{-}}\right)^{\top}\right)^{\top} \in$ $\mathcal{C}_{2 n}([-\tau, \tau])$ such that for all $t \in[-\tau, \tau]$,

$$
\phi_{z^{-}}(t) \leq x(t) \leq \phi_{z^{+}}(t) .
$$

Next, we introduce the notation

$$
\tilde{x}^{+}=x^{+}-x, \quad \tilde{x}^{-}=x-x^{-}, \quad \tilde{\nu}^{+}=\nu^{+}-\nu, \quad \tilde{\nu}^{-}=\nu-\nu^{-} .
$$

Then, using the fact that for all $t \geq \tau$, the solutions of the system (4.1) satisfy (4.12), we deduce that for all $t \geq \tau$,

$$
\left\{\begin{array}{l}
\dot{\tilde{x}}^{+}(t)=E_{P} \tilde{x}^{+}(t)-E^{-} \tilde{x}^{-}(t)+\rho_{1}(t)+\mathcal{P}_{1}(t), \\
\dot{\tilde{x}}^{-}(t)=E_{P} \tilde{x}^{-}(t)-E^{-} \tilde{x}^{+}(t)+\rho_{2}(t)+\mathcal{P}_{2}(t)
\end{array}\right.
$$

with

$$
\begin{aligned}
\mathcal{P}_{1}(t)= & w^{+}(t)-w^{-}(t)-B^{+}\left(W^{-}(t)-W(t)\right) \\
& -B^{-}\left(W^{+}(t)-W(t)\right) \\
\mathcal{P}_{2}(t)= & w(t)-w^{-}(t)+B^{+}\left(W^{+}(t)-W(t)\right) \\
& +B^{-}\left(W^{-}(t)-W(t)\right)
\end{aligned}
$$

and

$$
\begin{aligned}
& \rho_{1}(t)=G^{+} \tilde{\nu}^{+}(t)+H^{+} \tilde{\nu}^{+}(t-\tau)-G^{-} \tilde{\nu}^{-}(t)-H^{-} \tilde{\nu}^{-}(t-\tau), \\
& \rho_{2}(t)=G^{+} \tilde{\nu}^{-}(t)+H^{+} \tilde{\nu}^{-}(t-\tau)-G^{-} \tilde{\nu}^{+}(t)-H^{-} \tilde{\nu}^{+}(t-\tau) .
\end{aligned}
$$

Notice that Assumption 2 implies that for all $t \geq 0, \mathcal{P}_{1}(t) \geq 0, \mathcal{P}_{2}(t) \geq 0$. Next, observe that the matrix $E_{p}$ is cooperative and all the entries of the matrices $-E^{-}$,

Copyright (c) by SIAM. Unauthorized reproduction of this article is prohibited. 
$G^{+}, H^{+},-G^{-},-H^{-}$are nonnegative. It follows that Lemma B.1 in Appendix B applies to (4.17). On the other hand, (4.15) implies that $\left(\tilde{x}^{+}(t), \tilde{x}^{-}(t)\right)$ is a solution of the system (4.17) such that $\tilde{x}^{+}(t) \geq 0, \tilde{x}^{-}(t) \geq 0$ for all $t \in[-\tau, \tau]$. It follows that $\tilde{x}^{+}(t) \geq 0, \tilde{x}^{-}(t) \geq 0$ for all $t \geq-\tau$. This allows us to conclude that the system (4.14) is a 2 -framer for the system (4.1).

Now, we establish that Assumption 1 implies that the system (4.17) is exponentially stable when the disturbance $w$ is identically equal to zero and when $\tau$ satisfies (4.7). The system we consider is

$$
\left\{\begin{array}{l}
\dot{\tilde{x}}^{+}(t)=E_{P} \tilde{x}^{+}(t)-E^{-} \tilde{x}^{-}(t)+\rho_{1}(t), \\
\dot{\tilde{x}}^{-}(t)=E_{P} \tilde{x}^{-}(t)-E^{-} \tilde{x}^{+}(t)+\rho_{2}(t) .
\end{array}\right.
$$

We introduce the notation $\widetilde{X}=\left(\left(\tilde{x}^{+}\right)^{\top},\left(\tilde{x}^{-}\right)^{\top}\right)^{\top}$. The derivative of the positive definite quadratic function

$$
V(\widetilde{X})=\widetilde{X}^{\top} Q \widetilde{X}
$$

where $Q$ is the matrix satisfying (4.6) along the trajectories of (4.21), satisfies for all $t \geq \tau$,

$$
\dot{V} \leq-\|\tilde{X}(t)\|^{2}+2\|\tilde{X}(t)\| \cdot\|Q\| \cdot \sqrt{\left\|\rho_{1}(t)\right\|^{2}+\left\|\rho_{2}(t)\right\|^{2}},
$$

The triangle inequality implies that

$$
2|| \widetilde{X}(t)\|\cdot\| Q\left\|\cdot \sqrt{\left\|\rho_{1}(t)\right\|^{2}+\left\|\rho_{2}(t)\right\|^{2}} \leq \frac{1}{2}\right\| \widetilde{X}(t)\left\|^{2}+2\right\| Q \|^{2}\left[\left\|\rho_{1}(t)\right\|^{2}+\left\|\rho_{2}(t)\right\|^{2}\right] .
$$

It follows that that for all $t \geq \tau$,

$$
\dot{V} \leq-\frac{1}{2}\|\tilde{X}(t)\|^{2}+2\|Q\|^{2}\left[\left\|\rho_{1}(t)\right\|^{2}+\left\|\rho_{2}(t)\right\|^{2}\right] .
$$

Next, we observe that for all $(a, b, c, d) \in \Re^{4}$, the inequality $(a+b+c+d)^{2} \leq$ $4\left(a^{2}+b^{2}+c^{2}+d^{2}\right)$ is satisfied. From this inequality, we deduce that

$$
\begin{aligned}
\dot{V} \leq & -\frac{1}{2}\|\tilde{X}(t)\|^{2} \\
& +k\left[\left\|\tilde{\nu}^{+}(t)\right\|^{2}+\left\|\tilde{\nu}^{-}(t)\right\|^{2}+\left\|\tilde{\nu}^{+}(t-\tau)\right\|^{2}+\left\|\tilde{\nu}^{-}(t-\tau)\right\|^{2}\right]
\end{aligned}
$$

with

$$
k=16\|Q\|^{2} \max \left\{\left\|G^{+}\right\|,\left\|G^{-}\right\|,\left\|H^{+}\right\|,\left\|H^{-}\right\|\right\}^{2} .
$$

Using the Jensen inequality, one can prove that for all $t \geq 0$,

$$
\left\|\tilde{\nu}^{+}(t)\right\|^{2} \leq \tau \int_{t-\tau}^{t}\|\tilde{X}(\ell)\|^{2} d \ell,\left\|\tilde{\nu}^{-}(t)\right\|^{2} \leq \tau \int_{t-\tau}^{t}\|\tilde{X}(\ell)\|^{2} d \ell .
$$

It follows that for all $t \geq \tau$,

$$
\left\|\tilde{\nu}^{+}(t)\right\|^{2}+\left\|\tilde{\nu}^{-}(t)\right\|^{2}+\left\|\tilde{\nu}^{+}(t-\tau)\right\|^{2}+\left\|\tilde{\nu}^{-}(t-\tau)\right\|^{2} \leq 2 \tau \int_{t-2 \tau}^{t}\|\widetilde{X}(\ell)\|^{2} d \ell .
$$

Copyright $@$ by SIAM. Unauthorized reproduction of this article is prohibited. 
This inequality and (4.25) imply that for all $t \geq \tau$,

$$
\dot{V} \leq-\frac{1}{2}\|\widetilde{X}(t)\|^{2}+2 k \tau \int_{t-2 \tau}^{t}\|\widetilde{X}(\ell)\|^{2} d \ell .
$$

Consider now the Lyapunov-Krasovskii functional candidate:

$$
U(\phi)=V(\phi(0))+2 k \tau \int_{-2 \tau}^{0}\left(\int_{\ell}^{0}\|\phi(m)\|^{2} d m\right) d \ell
$$

Since $Q=Q^{\top}>0$, there exist constants $c_{1}, c_{2}>0$ such that for all $\phi \in \mathcal{C}_{2 n}([-2 \tau, 0])$,

$$
c_{1}\|\phi(0)\|^{2} \leq U(\phi) \leq c_{2} \sup _{\ell \in[-2 \tau, 0]}\|\phi(\ell)\|^{2} .
$$

The inequality (4.29) implies that the derivative of $U$ along the trajectories of (4.21) satisfies for all $t \geq 2 \tau$,

$$
\begin{aligned}
\dot{U} \leq & -\frac{1}{2}\|\tilde{X}(t)\|^{2}+2 k \tau \int_{t-2 \tau}^{t}\|\tilde{X}(\ell)\|^{2} d \ell-2 k \tau \int_{t-2 \tau}^{t}\|\tilde{X}(\ell)\|^{2} d \ell \\
& +4 k \tau^{2}\|\tilde{X}(t)\|^{2} \\
\leq & \left(-\frac{1}{2}+4 k \tau^{2}\right)\|\tilde{X}(t)\|^{2} .
\end{aligned}
$$

We now observe that $k \leq 16\|Q\|^{2} n^{4} p^{4}$, where $p$ is the constant defined in (4.2). It follows that if (4.7) is satisfied, then for all $t \geq 2 \tau$,

$$
\dot{U} \leq-\frac{1}{4}\|\tilde{X}(t)\|^{2}
$$

Finally, we can conclude the proof by invoking the Lyapunov-Krasovskii stability theorem [14].

4.3. Example. In this section, we apply Theorem 4.1 to the benchmark system (3.1). To begin, we check that this system satisfies Assumption 1. With the notation introduced in section 4.1, we have $A=0, B=-1, D=-1, E=E^{+}=E^{-}=0$, and $M=-I_{2}$. Therefore Assumption 1 is satisfied. Thus, Theorem 4.1 applies. By taking advantage of the proof of this theorem, we perform the construction of an exponential interval observer for (3.1) with $\tau \in\left[0, \frac{1}{8}\right]$.

Bearing in mind that for all $t \geq \tau$, the system (3.1) rewrites

$$
\dot{x}(t)=-x(t)-\int_{t-2 \tau}^{t-\tau} x(\ell) d \ell,
$$

we deduce from Theorem 4.1 that the dynamic extensions

$$
\left\{\begin{array}{l}
\dot{z}^{+}(t)=-z^{+}(t)-\int_{t-2 \tau}^{t-\tau} z^{-}(\ell) d \ell \\
\dot{z}^{-}(t)=-z^{-}(t)-\int_{t-2 \tau}^{t-\tau} z^{+}(\ell) d \ell
\end{array}\right.
$$

are a 2 -framer for (3.1). Using the equality $Q M+M^{\top} Q=-I_{2}$ with $Q=\frac{1}{2} I_{2}$, we deduce from (4.7) that (4.35) is exponentially stable when $\tau \in\left[0, \frac{1}{8}\right]$. The performance 


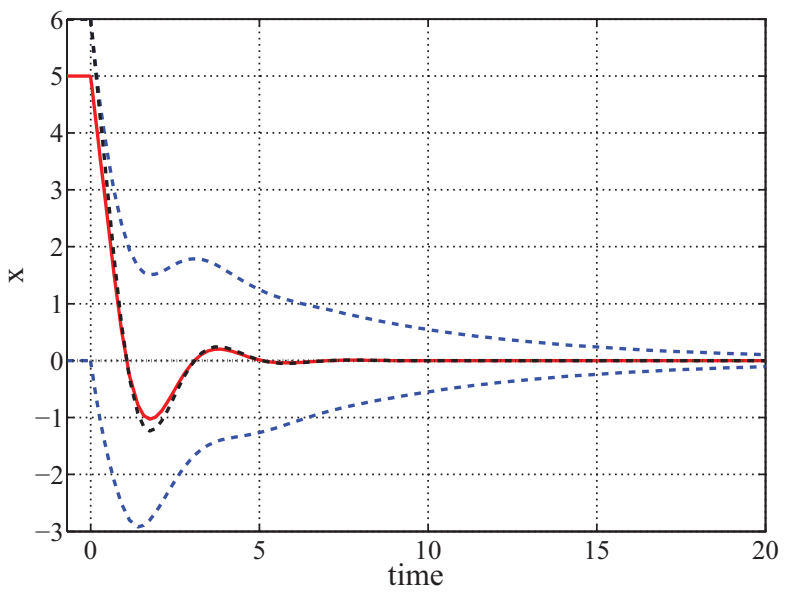

FIG. 4.1. Interval observer for the scalar system (3.1). The real trajectory is shown by a red continuous line. The "naive observer" (3.3) is shown in black dash dots or dots, and the proposed distributed delay interval observer (4.35) appears in dashed blue.

of this observer is illustrated in Figure 4.1 for a delay $\tau=0.1$. The figure shows also that the "naive" interval observer (3.3) is indeed not an interval observer since what was meant to be the upper bound can cross the real state and vice versa.

5. Application to a nonlinear model of bioreactor. An anaerobic digestion process used to produce methane from an organic pollutant $S \in[0,+\infty)$ using a bacterial species $X \in[0,+\infty)$ can be represented as follows:

$$
\left\{\begin{array}{l}
\dot{S}(t)=D\left[S_{i n}(t)-S(t)\right]-\frac{1}{Y} \mu(S(t)) X(t) \\
\dot{X}(t)=[\mu(S(t))-D] X(t)
\end{array}\right.
$$

with $D>0$ the dilution rate, $Y>0$ the yield coefficient, and $S_{i n}(t)>0$ the (unknown) substrate input concentration, possibly time varying. The growth rate $\mu(\cdot)$ is represented by a nonmonotonic function of Haldane type: $\mu(S)=a_{1} \frac{S}{S+a_{2}+S^{2} / a_{3}}$. (For more details, see [18].) This system is known to be bistable (for constant $S_{\text {in }}$ ) when $\sup _{S \geq 0} \mu(S)>D$ and $\arg \left(\sup _{S \geq 0} \mu(S)\right)<S_{i n}$ [31]. For this reason estimating the state variables is a key issue to be sure that the system is in the right working mode.

Without loss of generality, we can assume that $D=1$ and $Y=1$. Indeed, these constants can be removed through changes of variables, notations, and a timerescaling. The methane flow rate $y_{2}(t)=\mu(S(t)) X(t)$ can be on-line measured. Moreover, the substrate is off-line measured through complex procedures carried out by an operator. This involves a delay $\tau>0$ in the measurement, so that the available data is $y_{1}(t)=S(t-\tau)$.

We assume that

$$
S_{\text {in }}(t)=r+v(t),
$$

where $r$ is an unknown positive constant and $v(t)$ is an unknown Lipschitz continuous function such that there are known Lipschitz continuous functions $v^{-}, v^{+}$such that for all $t \geq-\tau$, the inequalities

$$
-\frac{r}{2} \leq v^{-}(t) \leq v(t) \leq v^{+}(t)
$$


are satisfied. Then (5.1) becomes

$$
\left\{\begin{aligned}
\dot{S}(t) & =r+v(t)-S(t)-\mu(S(t)) X(t) \\
\dot{X}(t) & =[\mu(S(t))-1] X(t)
\end{aligned}\right.
$$

The problem of estimating the influent substrate concentration, and especially $r$, is of key importance, together with the problem of state estimation. To solve this problem, we introduce the dynamic extension

$$
\left\{\begin{aligned}
\dot{\hat{S}}(t) & =\hat{r}(t)-\hat{S}(t)-y_{2}(t)+k_{1}\left[y_{1}(t)-\hat{S}(t-\tau)\right] \\
\dot{\hat{X}}(t) & =-\hat{X}(t)+y_{2}(t) \\
\dot{\hat{r}}(t) & =k_{2}\left[y_{1}(t)-\hat{S}(t-\tau)\right]
\end{aligned}\right.
$$

where $k_{1}, k_{2}$ are real numbers to be selected.

The dynamics of $\hat{X}$ lends itself to the construction of an exponentially stable interval observer. Hence, we focus on the two-dimensional system obtained setting $\bar{S}(t)=S(t)-\hat{S}(t)$ and $\bar{r}(t)=r-\hat{r}(t)$ :

$$
\left\{\begin{array}{l}
\dot{\bar{S}}(t)=\bar{r}(t)-\bar{S}(t)-k_{1} \bar{S}(t-\tau)+v(t) \\
\dot{\bar{r}}(t)=-k_{2} \bar{S}(t-\tau)
\end{array}\right.
$$

Choosing $k_{1}=k_{2}=1$ and introducing the variable

$$
q(t)=-\bar{S}(t)+\bar{r}(t)
$$

we obtain the system

$$
\left\{\begin{array}{l}
\dot{q}(t)=-q(t)-v(t) \\
\dot{\bar{r}}(t)=-\bar{r}(t-\tau)+q(t-\tau)
\end{array}\right.
$$

From Proposition 2.2, we deduce that this system is not nonnegative. From section 4.3 , we deduce that

$$
\left\{\begin{array}{l}
\dot{q}^{+}(t)=-q^{+}(t)-v^{-}(t), \\
\dot{\bar{r}}^{+}(t)=-\bar{r}^{+}(t)-\int_{t-2 \tau}^{t-\tau} \bar{r}^{-}(\ell) d \ell+\int_{t-2 \tau}^{t-\tau} q^{+}(\ell) d \ell+q^{+}(t-\tau), \\
\dot{q}^{-}(t)=-q^{-}(t)-v^{+}(t), \\
\dot{\bar{r}}^{-}(t)=-\bar{r}^{-}(t)-\int_{t-2 \tau}^{t-\tau} \bar{r}^{+}(\ell) d \ell+\int_{t-2 \tau}^{t-\tau} q^{-}(\ell) d \ell+q^{-}(t-\tau)
\end{array}\right.
$$

is an interval observer for (5.8) when $\tau \in\left[0, \frac{1}{8}\right]$. The state intervals are then given by

$$
\begin{aligned}
& \bar{r}^{-}(t)+\hat{r}(t) \leq r \leq \hat{r}(t)+\bar{r}^{+}(t), \\
& \bar{r}^{-}(t)-q^{+}(t)+\hat{S}(t) \leq S(t) \leq \hat{S}(t)+\bar{r}^{+}(t)-q^{-}(t) .
\end{aligned}
$$

The system (5.1) has been simulated with $S_{i n}(t)=10+3 \sin (t)$, bounding $v$ between $2 \sin (t)$ and $3 \sin (t)$. The delay was assumed to be $\tau=0.1$. The simulations presented in Figure 5.1 demonstrate the efficiency of the interval observer defined by (5.9) and (5.10). 

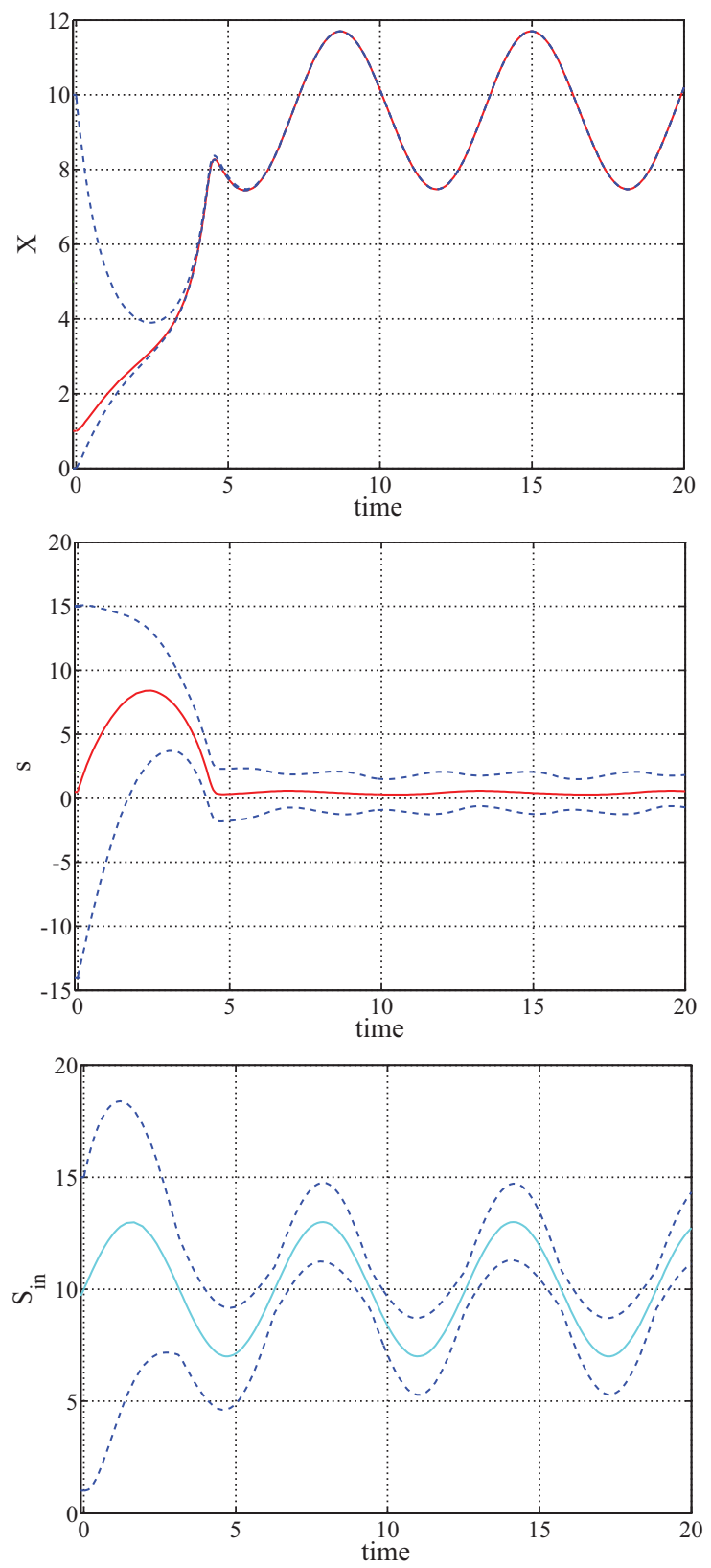

FIG. 5.1. Interval estimation performance in simulation. Real trajectories are shown by a red continuous line, input $S_{\text {in }}$ is a continuous light blue, and the interval observer appears in dashed dark blue.

6. Concluding remarks. Many extensions of the main results of our paper can be expected. Our results can be adapted to the case of systems endowed with inputs. Linear systems with multiple pointwise delays or distributed delays can be handled in a similar way as the linear systems with a single pointwise delay we have considered. Some extensions to nonlinear systems can also be expected. The open question we presented in section 3.2 about the existence of converging framers with pointwise delays for linear delay systems is also an interesting research subject.

Copyright $@$ by SIAM. Unauthorized reproduction of this article is prohibited. 
Appendix A. Proof of Proposition 3.2. Consider a 1-framer of the form (3.7). (We know that there exists at least one system of this type: the system (3.4).) Necessarily, $H_{1}$ and $H_{2}$ are linearly independent row vectors. It follows that there exists a change of coordinates which transforms the system (3.7) into

$$
\left\{\begin{aligned}
\dot{m}(t) & =R_{1,1} m(t)+R_{1,2} z(t)+S_{1,1} m(t-\tau)+S_{1,2} z(t-\tau), \\
\dot{z}(t) & =R_{2,1} m(t)+R_{2,2} z(t)+S_{2,1} m(t-\tau)+S_{2,2} z(t-\tau), \\
x^{+}(t) & =z^{+}(t), \\
x^{-}(t) & =z^{-}(t),
\end{aligned}\right.
$$

where $z(t)=\left(z^{+}(t), z^{-}(t)\right)^{\top} \in \Re^{2}$ and the matrices $R_{i, j}, S_{i, j}$ are matrices of appropriate dimension. Let $L_{1}, L_{2}$ denote the two rows of $R_{2,1}$ and assume that $L_{1} \neq 0$. Next, choose the initial conditions $\phi_{z}(t)=0, \phi_{x}(t)=0$ for all $t \in[-\tau, 0], \phi_{m}(0)=-L_{1}^{\top}$, $\phi_{m}(-\tau)=0$. Then $z^{+}(0)=x(0)$ and $\dot{z}^{+}(0)-\dot{x}(0)=-\left\|L_{1}\right\|^{2}<0$.

It follows that there exists an instant $t_{c}>0$ such that $z^{+}\left(t_{c}\right)<x\left(t_{c}\right)$. This yields a contradiction. We deduce that $L_{1}=0$. Similarly, one can prove that $L_{2}=0$ and therefore $R_{2,1}=0$. Arguing similarly, one can also prove that $S_{2,1}=0$. As an immediate consequence, we deduce that the $z$-subsystem of (A.1) simplifies as

$$
\dot{z}(t)=R_{2,2} z(t)+S_{2,2} z(t-\tau) .
$$

We rewrite this system

$$
\left\{\begin{array}{l}
\dot{z}^{+}(t)=a z^{+}(t)+b z^{-}(t)+e z^{+}(t-\tau)+f z^{-}(t-\tau), \\
\dot{z}^{-}(t)=c z^{+}(t)+d z^{-}(t)+g z^{+}(t-\tau)+h z^{-}(t-\tau) .
\end{array}\right.
$$

Next, choosing initial conditions $\phi_{x}, \phi_{z}$ such that $\phi_{x}(t)=\phi_{z^{+}}(t)=\phi_{z^{-}}(t)$ for all $t \in[-\tau, 0]$ and $\phi_{x}(-\tau)=0$, we obtain

$$
\left\{\begin{array}{l}
\dot{z}^{+}(0)-\dot{x}(0)=(a+b) \phi_{x}(0), \\
\dot{z}^{-}(0)-\dot{x}(0)=(c+d) \phi_{x}(0) .
\end{array}\right.
$$

If $a+b \neq 0$, then choosing $\phi_{x}(0)=-(a+b)$ we obtain $\dot{z}^{+}(0)-\dot{x}(0)=-(a+b)^{2}<0$. Arguing as we did to establish that $R_{2,1}=0$, we deduce that necessarily $a+b=0$. Similarly, one can prove that $c+d=0$.

Next, we choose initial conditions $\phi_{x}, \phi_{z}$ such that $\phi_{x}(t)=\phi_{z^{+}}(t)=\phi_{z^{-}}(t)$ for all $t \in[-\tau, 0]$ and $\phi_{x}(0)=0$. This choice gives

$$
\left\{\begin{array}{l}
\dot{z}^{+}(0)=(e+f) \phi_{x}(-\tau) \\
\dot{z}^{-}(0)=(g+h) \phi_{x}(-\tau) .
\end{array}\right.
$$

It follows that

$$
\left\{\begin{array}{l}
\dot{z}^{+}(0)-\dot{x}(0)=(e+f+1) \phi_{x}(-\tau) \\
\dot{x}(0)-\dot{z}^{-}(0)=(-g-h-1) \phi_{x}(-\tau) .
\end{array}\right.
$$

Arguing as we did above to establish that $a+b=0$, we can prove that necessarily $e+f=-1$ and $g+h=-1$. It follows that the system (A.3) rewrites

$$
\left\{\begin{array}{l}
\dot{z}^{+}(t)=a\left[z^{+}(t)-z^{-}(t)\right]+e z^{+}(t-\tau)-(1+e) z^{-}(t-\tau), \\
\dot{z}^{-}(t)=c\left[z^{+}(t)-z^{-}(t)\right]+g z^{+}(t-\tau)-(1+g) z^{-}(t-\tau) .
\end{array}\right.
$$

Copyright $@$ by SIAM. Unauthorized reproduction of this article is prohibited. 
Let $\tilde{z}^{+}=z^{+}-x, \tilde{z}^{-}=x-z^{-}$. Then

$$
\left\{\begin{aligned}
\dot{\tilde{z}}^{+}(t)= & a\left[z^{+}(t)-z^{-}(t)\right]+x(t-\tau)+e z^{+}(t-\tau) \\
& -(1+e) z^{-}(t-\tau), \\
\dot{\tilde{z}}^{-}(t)= & -x(t-\tau)+c\left[-z^{+}(t)+z^{-}(t)\right]-g z^{+}(t-\tau) \\
& +(1+g) z^{-}(t-\tau),
\end{aligned}\right.
$$

or, equivalently,

$$
\left\{\begin{array}{l}
\dot{\tilde{z}}^{+}(t)=a\left[\tilde{z}^{+}(t)+\tilde{z}^{-}(t)\right]+\tilde{z}^{-}(t-\tau)+e\left[\tilde{z}^{+}(t-\tau)+\tilde{z}^{-}(t-\tau)\right], \\
\dot{\tilde{z}}^{-}(t)=-c\left[\tilde{z}^{+}(t)+\tilde{z}^{-}(t)\right]-\tilde{z}^{-}(t-\tau)-g\left[\tilde{z}^{+}(t-\tau)+\tilde{z}^{-}(t-\tau)\right] .
\end{array}\right.
$$

From Proposition 2.2, we deduce that necessarily $a \geq 0, c \leq 0, e \geq 0, g \leq-1$. Besides, $y=\tilde{z}^{+}+\tilde{z}^{-}$satisfies

$$
\dot{y}(t)=(a-c) y(t)+(e-g) y(t-\tau) .
$$

Since $a-c \geq 0$ and $e-g \geq 1$, we deduce that (A.10) is exponentially unstable. It follows that (A.9) is not exponentially stable. Hence, there do not exist exponentially stable 1-framers of the form (3.7) for (3.1).

\section{Appendix B. Technical lemma.}

Lemma B.1. Let $T$ be a positive real number. Consider the system

$$
\dot{\chi}(t)=\mathcal{A} \chi(t)+\mathcal{B} \xi(t)+\mathcal{C} \xi(t-T)+P(t)
$$

with $\chi \in \Re^{N}, \xi(t)=\left(\xi_{1}(t), \ldots, \xi_{N}(t)\right)^{\top}$, and, for $i=1$ to $N, \xi_{i}(t)=\int_{t-T}^{t} \chi_{i}(\ell) d \ell$, where the matrix $\mathcal{A}$ is constant and cooperative, the matrices $\mathcal{B}$ and $\mathcal{C}$ are constant and with nonnegative entries, and the function $P$ is continuous and such that for all $t \geq 0, P(t) \geq 0$.

Let $\chi$ be a solution of (B.1) with the function $\phi_{\chi} \in \mathcal{C}_{N}([-T, T])$ as initial condition such that $\phi_{\chi}(t) \geq 0$ for all $t \in[-T, T]$. Then for all $t \in[-T,+\infty), \chi(t) \geq 0$.

Proof. To prove the result, we proceed by induction.

Induction hypothesis. The solution $\chi$ is such that $\chi(t) \geq 0$ for all $t \in[-T, k T]$, where $k \geq 1$ is an integer.

Step 1. Since $\chi(t)=\phi_{\chi}(t) \geq 0$ for all $t \in[-T, T]$, the induction assumption is satisfied at step 1 .

Step $j$. Assume that the induction hypothesis is satisfied at step $j \geq 1$. Then for all $t \in[j T,(j+1) T]$, we have $\xi(t-\tau) \geq 0$. It follows that for all $t \in[j T,(j+1) T]$, the function $\bar{P}(t)=\mathcal{C} \xi(t-\tau)+P(t)$ is nonnegative and we have

$$
\dot{\chi}(t)=\mathcal{A} \chi(t)+\mathcal{B} \xi(t)+\bar{P}(t) .
$$

Let, for $i=1$ to $N, \zeta_{i}(t)=\int_{j T}^{t} \chi_{i}(\ell) d \ell$ and $\zeta=\left(\zeta_{1}, \ldots, \zeta_{N}\right)^{\top}$. Then (B.2), in combination with the definition of $\zeta$, implies that for all $t \in[j T,(j+1) T]$,

$$
\left\{\begin{array}{l}
\dot{\chi}(t)=\mathcal{A} \chi(t)+\mathcal{B} \zeta(t)+\mathcal{B}[\xi(t)-\zeta(t)]+\bar{P}(t), \\
\dot{\zeta}(t)=\chi(t) .
\end{array}\right.
$$

Observe that for $i=1$ to $N$ and for all $t \in[j T,(j+1) T]$,

$$
\xi_{i}(t)-\zeta_{i}(t)=\int_{t-T}^{t} \chi_{i}(\ell) d \ell-\int_{j T}^{t} \chi_{i}(\ell) d \ell=\int_{t-T}^{j T} \chi_{i}(\ell) d \ell .
$$

Copyright $@$ by SIAM. Unauthorized reproduction of this article is prohibited. 
For all $t \in[j T,(j+1) T]$, we have $t-T \in[(j-1) T, j T]$, and therefore our induction assumption ensures that for all $m \in[t-T, j T], \chi(m) \geq 0$. We deduce that for all $t \in[j T,(j+1) T]$,

$$
\left\{\begin{array}{l}
\dot{\chi}(t)=\mathcal{A} \chi(t)+\mathcal{B} \zeta(t)+\widehat{P}(t) \\
\dot{\zeta}(t)=\chi(t)
\end{array}\right.
$$

where $\widehat{P}(t)=\mathcal{B}[\xi(t)-\zeta(t)]+\bar{P}(t)$ is a nonnegative function. Moreover, $\zeta(j T)=0$ and $\chi(j T) \geq 0$. In addition, since $\mathcal{A}$ is cooperative and the entries of $\mathcal{B}$ are nonnegative, the system (B.4) is cooperative and therefore its solutions with nonnegative values at the instant $j T$ are nonnegative over $[j T,(j+1) T]$. We immediately deduce that for all $t \in[j T,(j+1) T], \chi(t) \geq 0$. Thus, our induction assumption is satisfied at step $j+1$. This allows us to conclude.

\section{REFERENCES}

[1] O. Bernard And J.-L. GouzÉ, Closed loop observers bundle for uncertain biotechnological models, J. Process Control, 14 (2004), pp. 765-774.

[2] K.P.M. Bhat and H.N. Koivo, An observer theory for time-delay systems, IEEE Trans. Automat. Control, 21 (1976), pp. 266-269.

[3] M. Darouach, Linear functional observers for systems with delays in state variables, IEEE Trans. Automat. Control, 46 (2001), pp. 491-496.

[4] D. Dochain, Bioprocess Control, Wiley-ISTE, London, 2008.

[5] F.W. Fairman And A. Kumar, Delay-less observers for systems with delays, IEEE Trans. Automat. Control, 31 (1986), pp. 258-259.

[6] E. Fridman, New Lyapunov-Krasovskii functionals for stability of linear retarded and neutral type systems, Syst. Control Lett., 43 (2001), pp. 309-319.

[7] J.-L. GouzÉ, A. RAPAPORT, AND Z. HADJ-SADOK, Interval observers for uncertain biological systems, Ecological Model., 133 (2000), pp. 45-56.

[8] K. Gu, V.L. Kharitonov, and J. Chen, Stability of Time-Delay Systems, Birkhäuser, Boston, 2003.

[9] K. Gu AND S.I. Niculescu, Further remarks on additional dynamics in various model transformations of linear delay systems, IEEE Trans. Automat. Control, 46 (2001), pp. 497-500.

[10] J. Guckenheimer and P. Holmes, Nonlinear Oscillations, Dynamical Systems, and Bifurcations of Vector Fields, Appl. Math. Sci. 42, Springer-Verlag, New York, 1983.

[11] W.M. Haddad And V. Chellaboina, Stability theory for nonnegative and compartmental dynamical systems with time delay, Syst. Control Lett., 51 (2004), pp. 355-361.

[12] W.M. Haddad and V. Chellaboina, Nonnegative and Compartmental Dynamical Systems, Princeton University Press, Princeton, NJ, 2010.

[13] W.M. Haddad, V. Chellaboina, and T. Rajpurohit, Dissipativity theory for nonnegative and compartmental dynamical systems with time delay, IEEE Trans. Automat. Control, 49 (2004), pp. 747-751.

[14] J.K. Hale and S.M. Verduyn Lunel, Introduction to Functional Differential Equations, Appl. Math. Sci. 99, Springer, New York, 1993.

[15] L. Jaulin, M. Kieffer, O. Didrit, and E. Walter, Applied Interval Analysis, Springer, Berlin, 2001.

[16] M. Krstic, Delay Compensation for Nonlinear, Adaptive, and PDE Systems, Syst. Control Found. Appl., Birkhäuser, Boston, 2009.

[17] M. di Loreto, M. DaO, L. Jaulin, J.-F. Lafay, and J.-J. Loiseau, Applied interval computation: A new approach for time-delays systems analysis, in Applications of Time Delay Systems, J. Chiasson and J.-J. Loiseau, eds., Lecture Notes Control Inform. Sci. 352, Springer, London, pp. 175-197.

[18] L. Mailleret, O. Bernard, and J.-P. Steyer, Nonlinear adaptive control for bioreactors with unknown kinetics, Automatica, 40 (2004), pp. 1379-1385.

[19] F. MAZEnC And O. Bernard, Interval observers for linear time-invariant systems with disturbances, Automatica, 47 (2011), pp. 140-147.

[20] F. Mazenc and O. Bernard, Asymptotically stable interval observers for planar systems with complex poles, IEEE Trans. Automat. Control, 55 (2010), pp. 523-527.

Copyright $@$ by SIAM. Unauthorized reproduction of this article is prohibited. 
[21] F. Mazenc, S.I. Niculescu, And O. Bernard, Interval observers for linear systems with delay, in Proceedings of the 48th IEEE Conference on Decision and Control, Shanghai, China. 2009, pp. 1860-1865.

[22] W. Michiels and S.-I. Niculescu, Stability and Stabilization of Time-Delay Systems. An Eigenvalue Approach, SIAM, Philadelphia, 2007.

[23] M. MoISAn, Synthèse d'Observateurs par Intervalles pour des Systèmes Biologiques Mals Connus, Ph.D. thesis, Sophia-Antipolis, France, 2007.

[24] M. Moisan, O. Bernard, and J.-L. Gouzé, Near optimal interval observers bundle for uncertain bioreactors, Automatica, 45 (2009), pp. 291-295.

[25] M. MoISAN AND O. Bernard, Interval Observers for non-monotone systems. Application to bioprocess models, in Proceedings of the 16th IFAC World Congress, Prague, Czech Republic, 2005.

[26] S.-I. NiCulescu, Delay Effects on Stability. A Robust Control Approach, Lecture Notes Control Inform. Sci. 269, Springer, Heidelberg, 2001.

[27] A.E. Pearson and Y.A. Fiagbedzi, An observer for time-lag systems, IEEE Trans. Automat. Control, 34 (1989), pp. 775-777.

[28] A. RApaport And J.-L. Gouzé, Parallelotopic and practical observers for nonlinear uncertain systems, Internat. J. Control, 76 (2003), pp. 237-251.

[29] D. Salamon, Observers and duality between observation and state feedback for time delay systems, IEEE Trans. Automat. Control, 25 (1980), pp. 1187-1192.

[30] O. Sename, New trends in design of observers for time-delay systems, Kybernetika, 37 (2001), pp. $427-458$.

[31] H.L. Smith and P. Waltman, The Theory of the Chemostat, Cambridge University Press, Cambridge, UK, 1995.

Copyright (c) by SIAM. Unauthorized reproduction of this article is prohibited. 